\title{
Vehicle Routing Problem for perishable food in Cold Chain with Fuzzy Time Windows
}

\author{
Yanlun Tang and Guanwei Huang* \\ Sino-German School for Postgraduate Studies (CDHK), Tongji University, 200092 Shanghai, China \\ ${ }^{*}$ Corresponding author
}

\begin{abstract}
In this paper, a cold chain vehicle routing problem with fuzzy time windows (CVRPFTW) is proposed and solved. This paper applies fuzzy membership functions to characterize the customer's satisfaction level issues associated with time window violation in a vehicle routing problem and proposes CVRPFTW. CVRPFTW is formulated as a bi-objective model with two goals: (1) to minimize the total distribution cost and (2) to maximize the overall customer's satisfaction level. To solve this bi-objective model, a two-stage algorithm is developed. Using the two-stage algorithm, CVRPFTW is decomposed into two subproblems, namely a traditional vehicle routing problem with time windows for cold chain and a service improvement problem, and each of the objectives is sequentially solved. Then, aiming at the characteristics of the model and the practical problems in this paper, a genetic algorithm which is more suitable for this model is designed, which is called the improved genetic algorithm. Finally, experiments based on a real fresh food company are conducted, and the results show that the CVRPFTW model can achieve considerable cost-savings, while at the same time maintaining an acceptable customer's satisfaction level.
\end{abstract}

Keywords—cold chain logistics; Vehicle routing problem (VRP); fuzzy time windows; genetic algorithm

\section{INTRODUCTION}

The increasing trend for the fresh products in China raised various issued associated with quality, safety, and availability of these fresh products. Fresh foods are characterized by their perishable and temperature-sensitive nature. To make the fresh food available and achieve the quality and safety it requires proper cold chain management. Distribution planning is one of the major activities in cold chain management. Compared to normal temperature distribution, in addition to the extra investment in fleets of temperature-controlled vehicles, more controls of strict temperature and time are required to maintain food quality in cold chain distribution. However, this significantly increase the distribution cost. Furthermore, serving consumers within allowable delivery time windows and simultaneously meeting their expectation of food quality can increase the complexity of cold chain distribution planning [1]. As fresh food distribution companies tend to serve rather large numbers of customers in dispersed locations, it is crucial for them to solve the vehicle routing problem (VRP) in an efficient way so that to minimize the delivery cost and time while maintaining or even improving food and service quality for customers [2].

There has been little research on vehicle routing problem with fuzzy time windows (VRPFTW) adapted to cold chain logistics. Overall, two main directions have been observed from the existing research. One is that efforts have been made to consider the cost and impact of food quality deterioration in the planning models. The other is that VRPFTW has drawn attention. Few studies have addressed the issues which simultaneously involve these two directions. For the vehicle routing problem with fuzzy time windows, in which the fuzzy theory was applied, has begun to be considered. In practice, transportation usually involves routing vehicles according to customers' specific time windows, which are highly relevant to customers' satisfaction level. The vehicle routing problem with time windows (VRPTW) and its variations have been extensively studied by academic researchers, and a significant number of models and algorithms have been proposed $[3,4]$. Ever since Zadeh (1965) first proposed fuzzy theory, researchers from different areas have found fuzzy theory to be a useful tool to describe subjective opinions. In the field of network routing, there are few papers that successfully apply fuzzy theory to the time windows in routing problem $[5,6]$. In this paper, the problem of cold chain distribution planning will be modelled and solved, which aims to improve customer satisfaction levels within a fuzzy service time window at the lowest cold chain distribution cost.

Aiming at the optimization of the vehicle routing problem for cold chain logistics, which is to minimize the global distribution cost and to maximize the customer's satisfaction level, this paper proposes a scientific and effective bi-objective model for cold chain vehicle routing problem with fuzzy time windows (CVRPFTW).

The rest of this paper is organized as follows. Section 2 formulates CVRPFTW as a bi-objective programming problem and a two-stage algorithm is developed to decompose CVRPFTW into two subproblems and solve them sequentially; in Section 3, the solution of the model and the suitable genetic algorithm are presented; in Section 4, experimental results are analyzed, and Section 5 concludes this paper.

\section{MATHEMATICAL MODEL FOR CVRPFTW}

\section{A. Assumptions and Constraints}

Let $[\mathrm{N}, \mathrm{A}]$ be a symmetric directed network, where $\mathrm{N}=$ $\{0, \ldots, n\}$ is the set of nodes and $A=\{(i, j): i, j \in N\}$ is the set of arcs. Node 0 refers to the supplier, and nodes $1, \ldots, \mathrm{n}$ refer to the customers. Let $d_{i j}$ and $t_{i j}$ denote the distance and time associated with traveling from nodes $i$ to $j$ through arc $(i, j)$. Because the network is symmetric, $d_{i j}=d_{j i}$ and $t_{i j}=t_{j i}$. 
- This paper considers the case of one distribution centre with multiple customers;

- The distribution centre has a certain number of vehicles, and the specifications of the vehicles are the same and known;

- The customers need the products of the same temperature;

- The average daily demand of each customer for the goods $\mathrm{q}_{\mathrm{i}}$ is known;

- Link relation and distance among customers or customers with the distribution centre $d_{i j}$ are known;

- The vehicles moving velocity on average $\mathrm{V}$ is known and confirmed;

- The vehicle maintains a constant temperature difference between inside and outside $\Delta \mathrm{T}$ during distribution;

- In the distribution, the sum of demand capacity of all customers is not bigger than vehicle biggest load Q;

- Each customer is served only by one vehicle during distribution, but each vehicle can serve different customers;

- Each vehicle is send out by the distribution centre, and finally returns to the distribution centre;

- The service time can guarantee to meet the minimum satisfaction of each customer $\theta$.

\section{B. Model Formulation}

1) Customer's satisfaction level associated with time windows:

In a traditional VRPTW, a viable solution must meet all time windows, which is called hard time window. The customer's satisfaction level is determined by the actual arrival time, as shown in Figure 1.

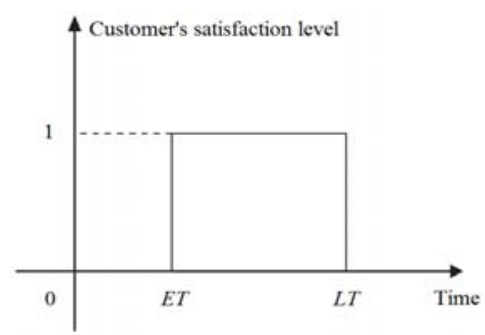
FIGURE I. THE SATISFACTION LEVEL FUNCTION OF A HARD TIME
WINDOW

It is noteworthy that if the customer isn't served within the scheduled time window, customer satisfaction will decline, but it does not mean that the supplier will lose this customer. There exist certain bounds on the violation (earliness or lateness) that a customer can endure. Each customer has a request for service time window [ET, LT]. When the service time falls within the time window [ET, LT], the customer's satisfaction level is good. But if the service time falls within the endurable time window [EET, ELT], the customer's satisfaction level could be determined by a certain fuzzy membership function. For problems with personal human emotions such as customer satisfaction, fuzzy set theory is a powerful tool. The reason why fuzzy time window is adopted in this paper is as follows: The general soft-time window problem, considering only minimizing the overall distribution costs, may sacrifice the delivery time and satisfaction level of some customers.

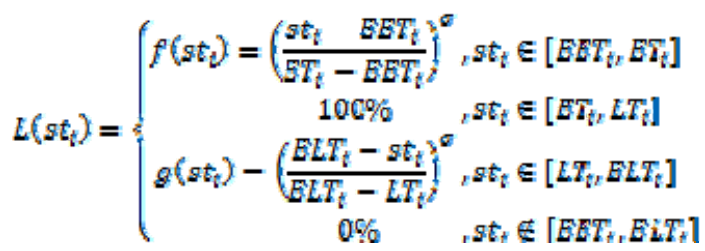

The service satisfaction is $\mathrm{L}, \mathrm{L}$ is between $[0,1]$. If the distribution service is completed in the time window, $\mathrm{L}=1$. The customer has an endurable time window between [EET, ELT]. Then can construct the fuzzy membership function like (1), where $\mathrm{st}_{\mathrm{i}}$ means the time to begin service for customer $\mathrm{i}$, and $\sigma$ denotes the coefficient of customer's sensitivity to time. With EET and ELT, the customer's satisfaction level can be described by a fuzzy membership function [7]. And the corresponding satisfaction level function can be described by Figure 2. Obviously, the earliness and lateness are closely related to the service quality of the supplier. The response of a customer satisfaction level to a given service time may not simply be "good" or "bad"; instead, it can be between "good" and "bad".

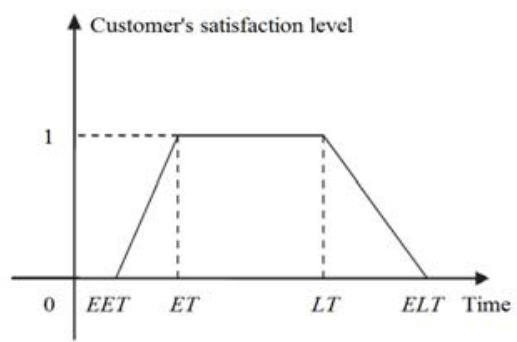

FIGURE II. THE SATISFACTION LEVEL FUNCTION OF FUZZY TIME WINDOWS

In this paper, to ensure that the customer will not be lost, the lowest customer's satisfaction level $\theta$ is assumed. With $L\left(s t_{t}\right) \geq \theta_{i}$, the corresponding actual acceptable time window $[\mathrm{e}, 1]$ can be derived as follows:

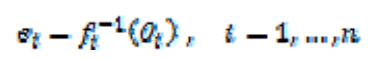

$$
l_{t}=g_{t}^{-1}\left(\theta_{t}\right), \quad t=1_{t=2} n
$$

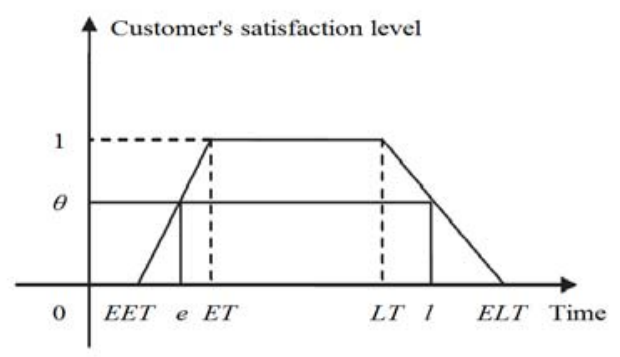

FIGURE III. LOWEST CUSTOMER'S SATISFACTION LEVEL $\Theta$ IN FUZZY TIME WINDOW

Due to the different amount of goods purchased by different customers, it is impossible to guarantee that each customer can 
achieve the greatest customer satisfaction. However, to maximize and rationalize the overall satisfaction $\mathrm{S}$, the importance of customer is appropriately divided in this model, according to the proportion of the total amount of goods purchased by the customers $\mathrm{q}_{\mathrm{i}}$ in the distribution plan:

$$
S=\frac{\sum_{t=:}^{n} L\left(s t_{t}\right) \cdot q_{t}}{\sum_{t+1}^{n} q_{t}}
$$

\section{2) Cold Chain Distribution Cost Analysis:}

a) The vehicle fixed cost $C_{l}$ : The fixed cost is mainly the depreciation cost of the vehicles and driver's wages $c_{0}$. It can be considered that it does not change with the distance of the customers:

$$
C_{1}=\sum_{k=1}^{m} \epsilon_{0}
$$

b) The vehicle transportation cost $C_{2}$ : The transportation cost $\mathrm{C}_{2}$ includes fuel cost, vehicle maintenance, etc. And the cost is generally proportional to the distance [8].

$$
C_{2}=\sum_{i=0}^{n} \sum_{i=1}^{n} \sum_{k=1}^{n} \epsilon_{d} d_{i j} X_{i j}^{k}
$$

Where $c_{d}$ denotes the unit transportation cost of the vehicles, and the decision variable $x_{t f}^{k}$ is defined as:

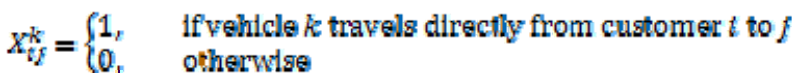

c) The cargo damage cost of frozen food $C_{3}$ : The main damage cost in the process of distribution consists of three parts: a) damage cost accumulated due to transit time, b) damage cost attribute to the waiting time for unloading after the vehicle already arrived and c) damage cost caused by door opening when servicing a customer will also accelerate spoilage including the arrival of food, unloading, code inspection and shelving [9]. We assume that the quality of the frozen food in the transportation process and customer service process is inversely proportional to the time and the demand of customers, respectively.

- The cargo damage cost during the transportation:

$$
C_{g_{a}}=p_{p} \cdot \sum_{k=1}^{m} \sum_{i=1}^{n} q_{i}^{k} \cdot a_{1} \cdot\left(t_{j}^{k}-t_{0}^{k}\right) \cdot Q_{t}
$$

where $p_{p}$ denotes the unit price of the products, $a_{1}$ denotes the cargo damage rate of the products during transportation, $\mathrm{t}^{\mathrm{k}}$ denotes the departure time of vehicle $\mathrm{k}$ from the distribution center, $\mathrm{t}^{\mathrm{k}}$ denotes the arrival time vehicle $\mathrm{k}$ at customer $\mathrm{i}, \mathrm{Q}_{\mathrm{i}}$ is the remaining demand of products when arrives at customer $i$ and the decision variable $y_{i}^{k}$ is defined as:

$$
Y_{F}^{k}= \begin{cases}1, & \text { if customer } f \text { is served by vehicle } k \\ 0 . & \text { otharwise }\end{cases}
$$

- The cargo damage cost during waiting for unloading:

$$
C_{9 b}=p_{p} \cdot \sum_{k=1}^{m} \sum_{i=1}^{n} \gamma_{f}^{k} \cdot \alpha_{2} \cdot \operatorname{Max}\left(0_{t} \theta_{t}-t_{t}\right) \cdot Q_{t}
$$

where $\mathrm{a}_{2}$ denotes the cargo damage rate of the products during waiting for unloading.

- The cargo damage cost during servicing:

$$
C_{2 \varepsilon}=p_{g} \cdot \sum_{k=1}^{m} \sum_{i=1}^{n} Y_{k}^{k} \cdot \alpha_{2} \cdot \frac{q_{t}}{\theta_{s}} \cdot Q_{t}
$$

where $\mathrm{a}_{3}$ denotes the cargo damage rate of the products during servicing, and $e_{\mathrm{s}}$ is the efficiency of service.

Hence, considering different damage rates and the price of the products, the cargo damage $\operatorname{cost} \mathrm{C}_{3}$ can be expressed by:

$$
C_{a}=C_{a x}+C_{a b}+C_{a b}
$$

d) The cooling cost $C_{4}$ : The cooling cost relates mainly to two aspects: One is heat transfer inside and outside the refrigerator caused by the temperature difference during transportation. On the other hand, heat will exchange caused by opening the door during loading/unloading process. The cooling cost can be obtained by calculating the cost of the refrigerant consumed. Consumption of the refrigerant is related to the heat transfer coefficient, the surface area of the vehicle, the outside temperature, stored product temperature and other factors.

a) The thermal load due to the difference in temperature $\Gamma_{\mathrm{t}}$ can be obtained by [10]:

$$
E_{t}=(1+a) \times \xi \times \sqrt{S_{\varepsilon} \times S_{i}} \times \Delta T
$$

where $\alpha$ is the depreciation degree of the vehicle, $\xi$ is the thermal conductivity, $S_{e}$ and $S_{i}$ are the external and internal surface area of the vehicle.

The cooling cost of refrigerator during the transportation is shown as follows:

$$
C_{4 \alpha}=\Gamma_{t} \cdot \sum_{i=0}^{n} \sum_{i=1}^{n} t_{t i} \cdot p_{c}
$$

where $\mathrm{p}_{\mathrm{c}}$ is the unit price of cooling.

The cooling cost of refrigerator during waiting for unloading can be expressed as follows: 


$$
C_{4 i}=\Gamma_{t} \cdot \sum_{i=n}^{n} \sum_{j=1}^{n} \operatorname{Max}\left(0, e_{i}-t_{t}\right) \cdot p_{0}
$$

b) The vehicle thermal load during loading/unloading $\Gamma_{\mathrm{o}}$ can be calculated by:

$$
E_{n}=(0.54 N+3.22) \times \Delta T \times \beta
$$

where $\beta$ is related to door opening frequency. Its value is shown in Table 1.

\section{TABLE I. CROSS-REFERENCING BETWEEN $\beta$ AND DOOR OPENING FREQUENCY}

\begin{tabular}{|c|c|c|}
\hline Degree & Door opening frequency & $\beta$ \\
\hline A & Closed & 0.25 \\
\hline B & Half of C & 0.5 \\
\hline C & $2-3$ times per hour & 1 \\
\hline D & More than $50 \%$ of C & 1.5 \\
\hline E & Double C & 2 \\
\hline
\end{tabular}

The cooling cost during servicing can be expressed by

$$
C_{4 e}=E_{e} \cdot \sum_{i=0}^{\mathrm{n}} q_{i} F \rho_{g} \cdot w_{2}
$$

The total energy consumption cost $C_{4}$ is the sum of $C_{4 a}, C_{4 b}$ and $C_{45}$ :

$$
C_{4}=\sum_{k=1}^{m}\left(C_{4 s}+C_{1 b}+C_{v e}\right)
$$

Therefore, the total cost for the vehicles to visit all customers is following:

$$
C=C_{1}+C_{2}+C_{8}+C_{4}
$$

\section{A Two-Stage Algorithm for CVRPFTW}

\section{1) $\quad$ VVRPTW- $\theta$ :}

The first stage of the algorithm is to minimize the distribution cost, and the second is to maximize the customer's satisfaction level. This order is adopted because an acceptable satisfaction level for each customer is guaranteed due to the service levels constraint. With $\theta$, what the supplier wants to achieve the most is to reduce the total cost, and then to further increase the service level. Therefore, for the bi-objective functions in this paper, the cost minimization function is the main objective function.

With (2) and (3), the earliest and latest service times that the customers can accept $\left[\mathrm{e}_{\mathrm{i}}, \mathrm{l}_{\mathrm{i}}\right]$ can be calculated. Therefore, the original CVRPFTW can be transformed into a cold chain vehicle routing problem with time windows (named as CVRPTW- $\theta$ ) by changing $\left[\mathrm{ET}_{\mathrm{i}}, \mathrm{LT}_{\mathrm{i}}\right]$ to $\left[\mathrm{e}_{\mathrm{i}}, \mathrm{l}_{\mathrm{i}}\right]$, and by removing the objective of maximizing the overall satisfaction level. This CVRPTW- $\theta$ can be solved using exact or heuristic algorithms.

\section{2) Service improvement problem:}

After CVRPTW- $\theta$ is solved, what remains is to improve the service level, and this problem can be named as "service improvement problem" (SIP). Through comparing the values of the cost function with different $\theta$, it can be determined whether it is worth raising a certain cost to achieve a higher level of customer satisfaction.

\section{3) The two-stage algorithm for CVRPFTW:}

Step 1: Calculate $\mathrm{e}_{\mathrm{i}}$ and $\mathrm{l}_{\mathrm{i}}$ with a given value of $\theta$ for each customer, using (2) and (3), respectively.

Step 2: With $\left[\mathrm{e}_{\mathrm{i}}, \mathrm{l}_{\mathrm{i}}\right]$ denoting the new time window, CVRPTW- $\theta$ is built and solved optimally using the genetic algorithm. The total distribution cost is minimized.

Step 3: Increase the value of $\theta$ gradually and record the minimum total cost values under different $\theta$.

Step 4: Compare the values of the cost function with different $\theta$ to determine whether it is worth raising a certain cost to achieve a higher level of customer satisfaction.

Step 5: Output total cost, $\theta$ and the corresponding routing solution as the final solution.

Therefore, the transferred model of CVRPTW- $\theta$ can be written as:

$$
\operatorname{Min} C
$$

$$
\sum_{i=1}^{n} \sum_{k=1}^{m} x_{i j}^{k} \leq m, \quad t=0
$$

$$
\sum_{i=1}^{n} X_{i f}^{k}=\sum_{i=1}^{n} X_{f i}^{k} \leq 1, \quad t=0, k=1,2, \ldots, m
$$

$$
\begin{aligned}
& \sum_{f=0}^{n} \sum_{k=1}^{m} Y_{t j}^{k}=1, \quad t=1,2, n_{r} n ; t \neq t \\
& \sum_{i=n}^{n} \sum_{k=1}^{m} X_{t j}^{k}=1, \quad f=1,2_{r+n_{r}} n n_{j} t \neq f \\
& \sum_{i=1}^{n} q_{i} \sum_{i=0}^{n} x_{i j}^{k} \leq q, \quad k=1,2, \ldots, m
\end{aligned}
$$

$$
\operatorname{Max}\left(a t_{t}, \theta_{t}\right) \leq s t_{t} \leq t_{t}
$$

Equation (18) is the objective of CVRPTW- $\theta$. Constraint (19) expresses that the number of paths must be less than or equal to the number of vehicles; Constraint (20) means that 
each vehicle is send out by the distribution center, and finally returns to the distribution center; Constraint (21) and (22) represent only single visits to each customer; (23) is the vehicle capacity constraint; (24) represents the constraint of service start time under fuzzy time window.

\section{IMPROVED GENETIC ALGORITHMS}

\section{A. Encoding}

This paper uses integer encoding. The chromosome code is shown in Figure 4 In the chromosome $C_{1}-C_{i}$ are the encodings of each customer by their service sequence, which are randomly generated integers. And $\mathrm{V}_{1}-\mathrm{V}_{\mathrm{k}}$ are the encodings of the vehicles, which represent the number of customers that each vehicle serves.

$$
\begin{aligned}
& \qquad\left(\mathrm{C}_{1}, \mathrm{C}_{2}, \ldots, \mathrm{C}_{\mathrm{i}}, 0, \mathrm{~V}_{1}, \mathrm{~V}_{2}, \ldots, \mathrm{V}_{\mathrm{k}}\right) \\
& \text { encodings of customer encodings of vehicles } \\
& \text { FIGURE IV. CHROMOSOME REPRESENTATION }
\end{aligned}
$$

\section{FIGURE IV. CHROMOSOME REPRESENTATION}

\section{B. Population Initialization}

In general, the generation of the initial population is taken in a random way, but this model with customer's fuzzy time window restrictions need to consider the actual feasible time windows calculated from the customer satisfaction level. Therefore, screening conditions are introduced during the population initialization, so that the initial populations are always feasible solution, which greatly reduces the number of iterations in the subsequent genetic operation and the running time of the program. Until a sufficient number of chromosomes are constructed that meet the requirements.

\section{Fitness Function}

Because the roulette wheel selection method is used in this model, the fitness function must be nonnegative, and the greater the value is, the better it is. Since the objective function of this model is to minimize the cost function, let the fitness function $\mathrm{F}$ be equal to the reciprocal of the objective function $\mathrm{f}$. So that the greater the value of the fitness function is, the more likely the chromosome is to enter the next generation.

$$
\text { Eitness }=1 \mathrm{ff}
$$

\section{Genetic Operation}

\section{1) Selection:}

In the roulette wheel selection, the fitness function assigns a fitness to possible solutions or chromosomes. This fitness level is used to associate a probability of selection with each individual chromosome. If $f_{i}$ is the fitness of individual $i$ in the population, its probability of being selected is $\frac{p_{i}}{p_{k}}-\frac{f_{k}}{\sum_{k+1} f_{b}}$, where $\mathrm{N}$ is the number of individuals in the population.

\section{2) Crossover:}

According to the encoding method and the characteristics of the initial population structure, it is determined that the crossover operation of this model is to crossover the coding of the customer's service sequence and the coding of the vehicle, to generate new population individuals. In this model, the uniform crossover method is used to crossover the vehicle code and the customer code. Uniform crossover operator decides (with a fixed mixing ratio between two parents) which parent will contribute how the gene values in the offspring chromosomes. Unlike single- and two-point crossover, the uniform crossover enables the parent chromosomes to contribute the gene level rather than the segment level.

Consider the two parents selected for crossover.

\begin{tabular}{|c|c|c|c|c|c|c|c|c|c|c|c|c|c|c|c|c|}
\hline Parent 1 & 1 & 1 & 0 & 1 & 1 & 0 & 0 & 1 & 0 & 0 & 1 & 1 & 0 & 1 & 1 & 0 \\
\hline Parent 2 & 1 & 1 & 0 & 1 & 1 & 1 & 1 & 0 & 0 & 0 & 0 & 1 & 1 & 1 & 1 & 0 \\
\hline
\end{tabular}

If the mixing ratio is 0.5 approximately, then half of the genes in the offspring will come from parent 1 and the other half will come from parent 2 . The possible set of offspring after uniform crossover would be as follows:

\begin{tabular}{|c|c|c|c|c|c|c|c|c|c|c|c|c|c|c|c|c|}
\hline $\begin{array}{c}\text { Offspring } \\
\mathbf{1}\end{array}$ & 1 & 1 & 0 & 1 & 1 & 1 & 1 & 0 & 0 & 0 & 0 & 1 & 1 & 1 & 1 & 0 \\
\hline $\begin{array}{c}\text { Offspring } \\
\mathbf{2}\end{array}$ & 1 & 1 & 0 & 1 & 1 & 0 & 0 & 1 & 0 & 0 & 1 & 1 & 0 & 1 & 1 & 0 \\
\hline
\end{tabular}
3) Mutation:

Mutation is a genetic operator used to maintain genetic diversity from one generation of a population of chromosomes to the next. Mutation occurs during evolution according to a defined mutation probability, usually set to a low value, such as 0.01. In this model, based on the encoding method of the chromosomes, the uniform mutation method is used. Therefore, the mutation operator replaces the value of the chosen gene with a uniform random value selected between the upper and lower bounds for the gene [11].

\section{EXPERIMENTS AND COMPUTATIONAL ANALYSIS}

For the computational experiments, we turn to a real company working in Shanghai. It is a multi-channel fresh food company with its own logistics network covering all districts in Shanghai. The improved genetic algorithm is realized by encoding in MATLAB software. We set the service level from the lowest $80 \%$ to the highest $100 \%$. All the tests are solved on a $2.30 \mathrm{GHz}$ Intel CPU in Microsoft Windows 10 with $8 \mathrm{~GB}$ memory. We ran the procedure for 10 times for each situation.

The experimental results are listed in Table 2

TABLE II. COMPARISON OF OBJECTIVE FUNCTION VALUES (DISTRIBUTION COSTS IN CNY) WITH DIFFERENT SATISFACTION LEVELS

\begin{tabular}{|c|c|c|c|c|c|}
\hline \multirow{2}{*}{$\#$} & \multicolumn{5}{|c|}{ Customer's satisfaction level } \\
\cline { 2 - 6 } & $\mathbf{8 0 \%}$ & $\mathbf{8 5 \%}$ & $\mathbf{9 0 \%}$ & $\mathbf{9 5 \%}$ & $\mathbf{1 0 0 \%}$ \\
\hline $\mathbf{1}$ & 6440.90 & 6621.65 & 6649.44 & 6741.87 & 7533.71 \\
\hline $\mathbf{2}$ & 6459.27 & 6542.13 & 6673.94 & 6826.34 & 7544.38 \\
\hline $\mathbf{3}$ & 6456.91 & 6586.45 & 6715.80 & 6760.38 & 7501.36 \\
\hline $\mathbf{4}$ & 6408.60 & 6558.75 & 6673.08 & 6774.76 & 7516.16 \\
\hline $\mathbf{5}$ & 6433.87 & 6549.86 & 6650.38 & 6759.17 & 7540.46 \\
\hline $\mathbf{6}$ & 6402.82 & 6585.89 & 6659.79 & 6757.29 & 7510.67 \\
\hline $\mathbf{7}$ & 6420.50 & 6518.43 & 6665.47 & 6781.21 & 7501.91 \\
\hline $\mathbf{8}$ & 6425.53 & 6576.37 & 6654.82 & 6743.96 & 7530.66 \\
\hline $\mathbf{9}$ & 6356.94 & 6588.48 & 6694.32 & 6791.06 & 7554.90 \\
\hline $\mathbf{1 0}$ & 6463.48 & 6546.62 & 6634.32 & 6791.98 & 7539.64 \\
\hline Average & 6426.88 & 6567.46 & 6667.14 & 6772.80 & 7527.38 \\
\hline
\end{tabular}


It can be seen from Figure 5 that with the same customer's overall satisfaction level the fluctuation of the objective function value is very small. When the overall satisfaction level of customers is between $80 \%$ and $95 \%$, the difference of the objective function value is relative tiny. That means when the customer's overall satisfaction level varies from $80 \%$ to $95 \%$, the total distribution costs doesn't increase remarkably. For each 5\% increase of customer's satisfaction level, the total distribution cost increases about $1.5 \%$ to $2.0 \%$. However, when customer's overall satisfaction level increased from $95 \%$ to $100 \%$, the total distribution costs increased by almost $12 \%$. As can be seen from this figure, when the customer's satisfaction level increases, the total distribution cost will increase accordingly. However, when the overall satisfaction of the customer is determined to be a certain value, although it increase s little distribution cost, it has a very significant impact on improving the overall customer satisfaction. Therefore, in the model of this case, we found that customer satisfaction level $\theta=95 \%$ is the most reasonable customer satisfaction level by comparison.

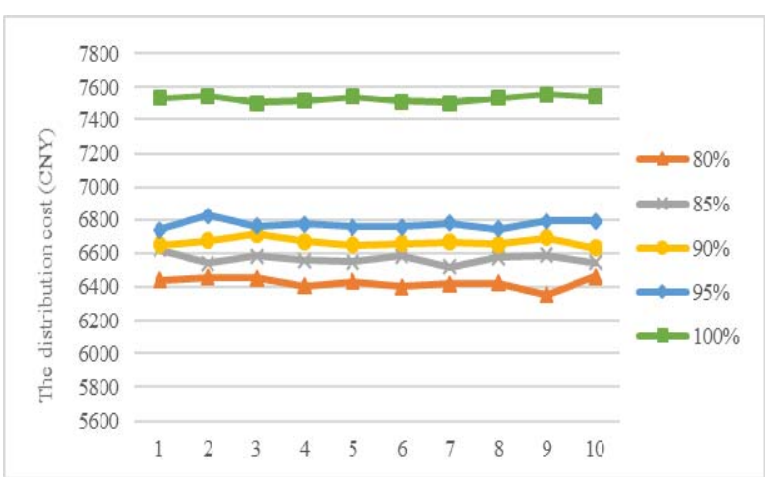

FIGURE V. COMPARISON OF OBJECTIVE FUNCTION VALUES WITH DIFFEREN SATISFACTION LEVELS

Through the above comparison, the overall customer satisfaction level can be set at $95 \%$, because in the $95 \%$ circumstance, it will not bring too high logistics cost for the company but can achieve the much better level of customer satisfaction, increasing the credibility of the company. If the company want to improve based on satisfaction of $95 \%$, it will bring about a sharp increase in cost. When the overall customer satisfaction level is set at $95 \%$, the best solution among the 10 times program running is shown in Table 3 as following:

TABLE III. OPTIMIZATION SOLUTION FOR THE COMPANY

\begin{tabular}{|c|c|c|}
\hline Vehicle No. & Route & $\begin{array}{c}\text { The distribution } \\
\text { cost (CNY) }\end{array}$ \\
\hline $\mathbf{1}$ & $0-8-22-13-23-24-26-0$ & \\
\hline $\mathbf{2}$ & $0-14-18-25-11-19-0$ & \\
\hline $\mathbf{3}$ & $0-29-27-2-1-7-10-0$ & \multirow{2}{*}{6741.9} \\
\hline $\mathbf{4}$ & $0-16-6-4-20-0$ & \\
\hline $\mathbf{5}$ & $0-21-28-5-0$ & \\
\hline $\mathbf{6}$ & $0-15-3-12-9-17-0$ & \\
\hline
\end{tabular}

After calculation, the comparison between the current routing plan and the optimized routing plan is as follows:
TABLE IV. COMPARISON OF CURRENT ROUTING PLAN AND THE OPTIMIZED ROUTING PLAN

\begin{tabular}{|l|c|c|}
\hline & $\begin{array}{c}\text { Current } \\
\text { routing plan }\end{array}$ & $\begin{array}{c}\text { Optimized } \\
\text { routing plan }\end{array}$ \\
\hline Total distance (km) & 724.5 & 584.4 \\
\hline Total delivery time (h) & 66.35 & 47.56 \\
\hline Fix cost (CNY) & 2600 & 1200 \\
\hline Transportation cost (CNY) & 1104.5 & 1558.8 \\
\hline Cooling cost (CNY) & 0 & 2298.1 \\
\hline Damage cost (CNY) & 9163.2 & 1684.9 \\
\hline Total delivery cost (CNY) & 12867.7 & 6741.9 \\
\hline
\end{tabular}

By comparison, we can find that the optimized routing plan has a great improvement in total delivery distance, total delivery time and total delivery cost. With the new routing plan, the company can save $140.1 \mathrm{~km}$ delivery distance, and nearly 20 hours delivery time per day. The total delivery cost can be saved daily $12867.7-6741.9=6125.8 \mathrm{CNY}$, which is nearly half of the current total delivery cost. Therefore, such improvement is very necessary for the company.

\section{CONCLUSION}

This paper studies a vehicle routing problem with fuzzy time windows in cold chain. Based on the concept of fuzzy time windows, CVRPFTW is formulated as a bi-objective problem with two objectives: minimizing the total cost and maximizing the overall customer's satisfaction level. A twostage algorithm is developed to decompose the original problem into two subproblems, and sequentially solves these objectives to optimization (total distribution cost first, satisfaction levels second). Based on the characteristics of this model, the genetic algorithm is improved. The computational experiments were designed based on actual information in a multi-channel fresh food company to verify the rationality and the feasibility of the model in improving the efficiency of distribution and customer satisfaction while ensuring the food fresh and safe.

This paper established a CVRPFTW model for the agricultural product with single temperature requirement. Therefore, the multi-temperature joint distribution with mixedtype vehicles will be the direction of future research.

\section{REFERENCES}

[1] H.K. Chen, C.F. Hsueh, M.S. Chang, "Production scheduling and vehicle routing with time windows for perishable food products," in Computers \& Operations Research 36 (7), 2009, pp. 2311-2319.

[2] Y. Zhang, X.D. Chen, "An Optimization Model for the Vehicle Routing Problem in Multi-product Frozen Food Delivery," in Journal of Applied Research and Technology 12 (2), 2014, pp. 239-250.

[3] Y.F. Deng, J.L.Xiang, Z.L.Ou, "Improvement of Genetic Algorithm for Vehicle Routing Problems with Time Windows," in : 2013 Third International Conference on Intelligent System Design and Engineering Applications. 2013 Third International Conference on Intelligent System Design and Engineering Applications (ISDEA). China, Hong Kong: IEEE, 2013, pp. 866-869.

[4] S. Iqbal, M. Kaykobad, M.S. Rahman, "Solving the multi-objective Vehicle Routing Problem with Soft Time Windows with the help of bees," in Swarm and Evolutionary Computation 24, 2015, pp. 50-64.

[5] S.F. Ghannadpour, S. Noori, R.Tavakkoli-Moghaddam, K. Ghoseiri, "A multi-objective dynamic vehicle routing problem with fuzzy time windows. Model, solution and application," in Applied Soft Computing 14, 2014, pp. 504-527. 
[6] D. Chen, E. Cao, W.T. Lai, "A differential evolution algorithm for pickups and deliveries problem with fuzzy time windows," in IFS 30 (1), 2015, pp. 267-277.

[7] J.F. Tang, Z.D. Pan, R.Y.K. Fung, H. Lau, "Vehicle routing problem with fuzzy time windows," in Fuzzy Sets and Systems 160 (5), 2009, pp. 683-695.

[8] H. Gu et al. "Optimization and algorithm for the vehicle distribution route of urban cold-chain logistics model," 3rd International Conference on Operations and Supply Chain Management. Wuhan, China, 2009.

[9] G. Zhao, Y.F. Zhang, "On the cold chain logistics routing optimization," in 30th Chinese Control Conference. Yantai, China, 2011.

[10] H.L. Wang, Y. Wang,Y.C. Zeng, "Distribution of perishable food based on models with time windows", in Industrial Engineering Journal 11 (3), 2008, pp. 127-130.

[11] D. Jaeger, R. Jung, Encyclopedia of Computational Neuroscience. New York, NY: Springer New York, 2015. 